\title{
Reference Result Target Anatomic Site Laterality Code
}

National Cancer Institute

\section{Source}

National Cancer Institute. Reference Result Target Anatomic Site Laterality Code. NCI

Thesaurus. Code C94040.

A coded value specifying the side of the body (or a paired organ) that is a target site for a result. 